\title{
The change of adherence to a process quality-of-care-indicator after the initiation of clinical audit in Korea
}

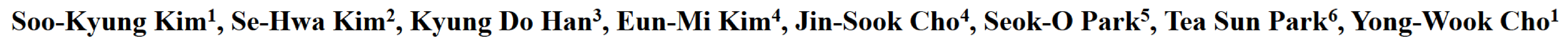

${ }^{1}$ Department of Internal Medicine, CHA Bundang Medical Center, CHA University, Seongnam, Korea; ${ }^{2}$ Department of Internal Medicine, Catholic Kwandong University, Incheon, Korea; ${ }^{3}$ Department of Biostatistics, College of Medicine, The Catholic University of Korea, Seoul, Korea; ${ }^{4}$ Health Insurance Review \& Assessment Service, Wonju, Korea;

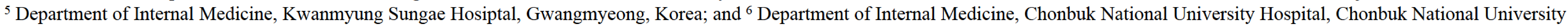
Medical School, Jeonju, Korea

\begin{abstract}
$\underline{\text { Abstract }}$
Background: Adherence to recommended guideline for monitoring of glucose control and screening of diabetic complication has been demonstrated to prevent the chronic complications of diabetes. In Korea, the clinical audit of quality of diabetes care has been started in 2011. The aim of this study is to investigate the change of adherence to a process quality-of-care-indicator in diabetes after the initiation of audit in Korea.

Methods: This retrospective study was performed using the national health insurance claims database from the Health Insurance Review \& Assessment Service (HIRA) of Korea, from 2009 to 2014. Study patients were aged 30 years or older, had type 2 diabetes, had taking at least one hypoglycemic agent in only one attending clinic at 2009, and had no history of any diabetes-related chronic complications including cardiovascular diseases before December, 2010, cancer, or $\geq 90$ days of admission $(\mathrm{n}=280,698)$. Process indicators included measurement of $\mathrm{HbAlc}$ more than once a year, annual measurement of lipid, annual examination for retinopathy and nephropathy.

Results: The number of patients receiving HbAlc and lipid measurement at least once a year increased to 69.9 and $54.5 \%$ in 2014 from 52.7 and $32.5 \%$ in 2009 . Although the annual screening of nephropathy and retinopathy is also increased gradually, it is still low at 16.5 and $24.5 \%$ in 2014. Despite a gradual increase in the proportion of patients who underwent annual measurement of HbAlc and lipid and annual screening for retinopathy and nephropathy, only $6.1 \%$ of the patients met all four criteria in 2014 (1.5\% in 2009 and $3.9 \%$ in 2011).

Conclusion: Although there were improvements in adherence to each process quality-of-care indicator, the percentage of patients undergoing all four examinations annually remained still very low.
\end{abstract}

Table 1. Percentage of patients receiving HIRA recommended care processes and state prescribing OHA by audit year

\begin{tabular}{|c|c|c|c|c|c|c|}
\hline & 2009 & 2010 & 2011 & 2012 & 2013 & 2014 \\
\hline $\mathrm{N}$ & 280,698 & 280,698 & 279,295 & 277,617 & 276,048 & 274,393 \\
\hline HbA1c measurement, more than once a year $(\mathrm{N},[\%])$ & $147,911(52.7)$ & $143,212(51.0)$ & $171,279(61.3)$ & $179,382(64.6)$ & $185,967(67.4)$ & $191,868(69.9)$ \\
\hline $\mathrm{HbA} 1 \mathrm{c}$ measurement, at least twice a year $(\mathrm{N},[\%])$ & $61,780(22.0)$ & $67,261(24.0)$ & $79,255(28.4)$ & $89,194(32.1)$ & $97,442(35.3)$ & $102,409(37.3)$ \\
\hline Lipid measurement, more than once a year $(\mathrm{N},[\%])$ & $91,329(32.5)$ & $91,722(32.7)$ & $110,723(39.6)$ & $119,631(43.1)$ & $126,988(46.0)$ & $149,417(54.5)$ \\
\hline Measurement of urinary albumin excretion (UAE), more than once a year $(\mathrm{N},[\%])$ & $13,744(4.9)$ & $15,255(5.4)$ & $26,802(9.6)$ & $32,989(11.9)$ & $39,388(14.3)$ & $45,241(16.5)$ \\
\hline Ophthalmologic examination, more than once a year $(\mathrm{N},[\%])$ & $36,596(13.0)$ & $28,247(13.6)$ & $56,575(20.3)$ & $59,181(21.3)$ & $64,592(23.4)$ & $67,157(24.5)$ \\
\hline $\operatorname{HbA} 1 \mathrm{c}+\operatorname{Lipid}(\mathrm{N},[\%])^{*}$ & $84,119(30.0)$ & $85,482(30.5)$ & $105,227(37.7)$ & $114,411(41.2)$ & $122,350(44.3)$ & $144,110(52.5)$ \\
\hline HbAlc + Lipid + UAE (N, [\%])* & $12,111(4.3)$ & $13,588(4.8)$ & 23,934 (8.6) & $29,074(10.5)$ & $35,354(12.8)$ & $42,022(15.3)$ \\
\hline $\mathrm{HbA} 1 \mathrm{c}+$ Lipid + Ophthalmologic examination $(\mathrm{N},[\%])^{*}$ & $15,114(5.4)$ & $16,025(5.7)$ & $28,764(10.3)$ & $31,957(11.5)$ & $36,389(12.2)$ & $42,882(15.6)$ \\
\hline HbA1c + Lipid + UAE + Ophthalmologic examination $(\mathrm{N},[\%])^{*}$ & $4,287(1.5)$ & $4,475(1.6)$ & $10,810(3.9)$ & $12,122(4.4)$ & $14,728(5.3)$ & $16,858(6.1)$ \\
\hline \multicolumn{7}{|l|}{ Oral hypogycemic agent (OHA) possession ratio $\geq 80 \%$} \\
\hline Total & $169,146(60.3)$ & $188,736(67.2)$ & $194,501(69.6)$ & $199,322(71.8)$ & $202,437(73.3)$ & $201,626(73.5)$ \\
\hline Tertiary general hospital & $13,383(66.6)$ & $14,010(74.9)$ & $13,868(73.9)$ & $13,548(77.1)$ & $14,345(80.9)$ & $14,662(80.3)$ \\
\hline General hospital & $12,004(54.2)$ & $13,635(68.8)$ & $14,378(71.3)$ & $15,851(76.1)$ & $17,530(77.8)$ & $19,061(78.2)$ \\
\hline Hospital & $8,756(45.9)$ & $10,213(61.9)$ & $10,520(66.5)$ & $11,279(73.0)$ & $11,208(74.5)$ & $11,020(74.6)$ \\
\hline Private clinic & $135,003(61.6)$ & $150,878(72.3)$ & $155,735(75.8)$ & $158,644(78.0)$ & $159,354(79.6)$ & $156,883(80.1)$ \\
\hline \multicolumn{7}{|l|}{ Quality of care $(\mathrm{N},[\%])$} \\
\hline \multicolumn{7}{|l|}{ Best $^{* *}$} \\
\hline Total & $2,926(1.0)$ & $3,506(1.3)$ & $8,099(2.9)$ & $9,545(3.4)$ & $12,054(4.4)$ & $13,670(5.0)$ \\
\hline Tertiary general hospital & $2,174(10.8)$ & $2,272(12.2)$ & $4,239(22.6)$ & $3,736(21.3)$ & $4,387(24.8)$ & $4,345(23.8)$ \\
\hline General hospital & $372(1.7)$ & $542(2.7)$ & $1,456(7.2)$ & $2,113(10.1)$ & $2,767(12.3)$ & $3,113(12.8)$ \\
\hline Hospital & $36(0.2)$ & $63(0.4)$ & $196(1.2)$ & $314(2.0)$ & $426(2.8)$ & $440(3.0)$ \\
\hline Private clinic & $344(0.2)$ & $629(0.3)$ & $2,208(1.1)$ & $3,382(1.7)$ & $4,474(2.2)$ & $5,772(3.0)$ \\
\hline \multicolumn{7}{|l|}{$\operatorname{Good}^{\dagger}$} \\
\hline Total & $52,629(18.8)$ & $63,348(22.6)$ & $79,149(28.3)$ & $88,695(32.0)$ & $96,783(35.1)$ & $114,316(41.7)$ \\
\hline Tertiary general hospital & $10,020(49.8)$ & $10,473(56.0)$ & $10,917(58.2)$ & $10,950(62.3)$ & $11,639(65.7)$ & $12,179(66.7)$ \\
\hline General hospital & $5,861(26.4)$ & $6,874(34.7)$ & $8,447(41.9)$ & $9,997(48.0)$ & $11,744(52.1)$ & $13,419(55.0)$ \\
\hline Hospital & $2,617(13.7)$ & $3,402(20.6)$ & $4,280(27.1)$ & $5,023(32.5)$ & $5,519(36.7)$ & $6,306(42.7)$ \\
\hline Private clinic & $34,131(15.6)$ & $42,599(20.4)$ & $55,505(27.0)$ & $67,725(30.8)$ & $67,881(33.9)$ & $82,412(42.1)$ \\
\hline
\end{tabular}

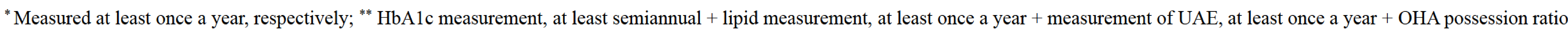
$\geq 80 \%$; ${ }^{\dagger} \mathrm{HbA} 1 \mathrm{c}$ measurement, at least once a year + lipid measurement, at least once a year + OHA possession ratio $\geq 80 \%$. 